\title{
A Influência de Variáveis Cognitivas e do TDAH na Leitura de Crianças
}

\author{
Juliana Campos Schmitt ${ }^{1, *}$ @ \& Francis Ricardo dos Reis Justi ${ }^{1}$ (1) \\ Universidade Federal de Juiz de Fora, Juiz de Fora, MG, Brasil
}

\begin{abstract}
RESUMO - O estudo investigou a influência de variáveis cognitivas na leitura de crianças com desenvolvimento típico e em crianças com Transtorno de Déficit de Atenção e Hiperatividade (TDAH). Participaram 70 crianças do $2^{\circ}$ ao $7^{\circ}$ anos do Ensino Fundamental, divididas nos grupos: TDAH e controle. Foram aplicadas tarefas de consciência fonológica, memória de trabalho fonológica, nomeação seriada rápida, vocabulário, quociente de inteligência (QI), atenção, flexibilidade cognitiva e leitura (precisão, fluência e compreensão). Análises de regressão linear múltipla indicaram que a nomeação seriada rápida e a consciência fonológica contribuíram fortemente para a precisão, a fluência e a compreensão de leitura. O TDAH influenciou somente na compreensão. Argumentou-se que provavelmente os componentes da função executiva são os responsáveis pela relação entre TDAH e compreensão de leitura.
\end{abstract}

PALAVRAS-CHAVE: TDAH, leitura, função executiva

\section{The Effects of Cognitive Variables and ADHD on Reading}

\begin{abstract}
This study investigated the influence of cognitive variables on reading in children with Attention Deficit Hyperactivity Disorder (ADHD) and children with typical development. Seventy children from 2th to 7th grade took part in this study and were divided into two groups: ADHD and control. Tasks of phonological awareness, phonological work memory, rapid automatized naming, vocabulary, intelligence quotient (IQ), attention, cognitive flexibility and reading (accuracy, fluency, and comprehension) were applied. Multiple linear regression analyses indicated that rapid serial naming and phonological awareness strongly contributed to reading accuracy, reading fluency, and comprehension. ADHD only influenced comprehension, probably because of its relation to executive function.
\end{abstract}

KEYWORDS: ADHD, reading, executive function

OTDAH é um transtorno do desenvolvimento que envolve o autocontrole e consiste em problemas com a manutenção da atenção, o controle de impulsos e o nível de atividade física e mental (Barkley, 2002). As crianças com TDAH apresentam alterações na função executiva que envolve processos mentais de inibição de resposta, de autorregulação do comportamento, entre outros (Barkley, 2005).

O diagnóstico do TDAH é fundamentalmente clínico, com apoio nos critérios sintomatológicos do DSM-5 (American Psychiatric Association, 2014) e na repercussão dos sintomas comportamentais na rede relacional e na vida escolar do indivíduo. $\mathrm{O}$ transtorno se caracteriza por uma combinação de dois grupos de sintomas: desatenção e hiperatividade/impulsividade. Esses grupos devem estar presentes no indivíduo por pelo menos seis meses e ocorrer em dois contextos diferentes, no mínimo. De acordo com o DSM-5, o TDAH se subdivide em três fenótipos comportamentais, intitulados especificadores de apresentação: TDAH com predomínio em desatenção (se o critério desatenção é preenchido, mas o critério de hiperatividade/impulsividade não é preenchido nos últimos seis meses), TDAH com predomínio de sintomas de hiperatividade/impulsividade (se o critério para hiperatividade/impulsividade é preenchido, mas o critério desatenção não é preenchido nos últimos seis meses) e TDAH combinado (se tanto o critério de desatenção quanto o de hiperatividade/impulsividade são preenchidos nos últimos seis meses).

De acordo com Phelan (2005), as estatísticas a respeito do TDAH indicam que há aproximadamente 1 criança com

\footnotetext{
*E-mail: julianaschmittufjf@hotmail.com

- Submetido: 07/04/2017; Revisado: 06/06/2019; Aceito: 19/04/2020.
} 
este transtorno em cada sala de aula com 20 a 25 alunos. A literatura (Cantwell \& Baker, 1991; Pastor \& Reuben, 2002) aponta que até $80 \%$ das crianças com TDAH exibem problemas de aprendizagem e/ou desempenho acadêmico. $\mathrm{O}$ transtorno é fator de risco para o mau desempenho escolar (Pastura et al., 2005), que pode ser observado quando o indivíduo apresenta rendimento acadêmico e habilidades cognitivas inferiores ao esperado para determinada idade $\mathrm{e}$ escolaridade (Fonseca, 2008). É recorrente o comportamento de crianças com o transtorno passar despercebido pelos pais. A maioria dos casos de TDAH é detectado na escola, pois é comum o indivíduo com TDAH apresentar dificuldades em memorizar e recordar informações já aprendidas; ser facilmente distraído da tarefa realizada devido aos estímulos irrelevantes (desatenção); ter dificuldade em permanecer quieto e esperar sua vez para falar; falar demais e interromper os colegas e professores (Barkley, 2002). De acordo com Coelho e Barbosa (2012), o custo social do TDAH não tratado ao longo da vida é alto, incluindo baixo aproveitamento escolar, repetências, expulsões e evasão escolar.

O TDAH vem sendo considerado preocupante pelos educadores no período em que a criança inicia seu contato com a leitura e escrita (Seno, 2010) já que, para que os objetivos pedagógicos propostos sejam alcançados, é necessário manter a atenção e a concentração sustentadas. Um estudo que investigou a relação entre TDAH e o desempenho na leitura em crianças foi o de Fergusson e Horwood (1992). O desempenho de leitura e os comportamentos relacionados ao TDAH foram avaliados quando os participantes estavam com 10 e 12 anos de idade. A técnica de modelagem de equação estrutural foi empregada e observou-se que o grau de deficit de atenção de uma criança de 12 anos influenciava negativamente no desempenho na leitura, embora não tenham sido apresentadas evidências que indicassem que a capacidade de leitura nessa idade exercia algum efeito sobre os deficit de atenção. Portanto, pelo menos em parte, os sintomas de TDAH parecem estar causalmente relacionados aos níveis de desempenho na leitura. Além disso, um estudo recente realizado por Miranda et al. (2017) traz indícios de que o pior desempenho em leitura de pessoas com TDAH pode ser observado até na vida adulta.

Tendo em vista as consequências que o TDAH pode acarretar na trajetória escolar do indivíduo e como a leitura é uma habilidade fundamental para o bom desempenho acadêmico, é importante conhecer, de forma específica, qual a relação entre o TDAH e o desempenho na leitura. De acordo com Grégoire e Pierart (1997), a competência em leitura pressupõe dois componentes: a precisão e a rapidez no reconhecimento das palavras (fluência); e as capacidades cognitivas e linguísticas necessárias para compreender uma mensagem escrita. Ademais, a identificação das palavras é condição necessária à leitura, pois não existe um bom leitor que seja deficiente no nível dos processos de identificação das palavras (Grégoire \& Pierart, 1997). Dentre os processos cognitivos e habilidades metalinguísticas importantes para a aprendizagem da leitura, destacam-se a consciência fonológica, a memória de trabalho fonológica e a nomeação seriada rápida (Justi \& Roazzi, 2012; Puliezi \& Maluf, 2012).

A consciência fonológica $(\mathrm{CF})$ pode ser definida como o conhecimento das subunidades que compõem a palavra falada e a habilidade de manipulação delas (Bradley \& Bryant,1983; Morais, 2005; Mousinho, 2003). É uma habilidade de natureza metacognitiva, visto que é necessário refletir acerca da estrutura fonológica da linguagem oral (Gombert, 1992). A CF desempenha um papel importante na aprendizagem da leitura e da escrita (Bradley \& Bryant, 1983; Wagner \& Torgesen, 1987), mas o aprendizado da leitura e da escrita também influencia no desenvolvimento das habilidades fonológicas (Morais et al., 1979). Leitores considerados habilidosos são aqueles que apresentam melhor desempenho em tarefas de CF (Goswami \& Bryant, 1990; Holligan \& Johnston, 1988).

Estudos (por exemplo, Cunha et al., 2013; Fragoso et al., 2013) mostram que as crianças que apresentam diagnóstico de TDAH, na maioria dos casos, têm a CF prejudicada e apresentam desempenho inferior em relação ao grupo controle (GC). Cunha et al. (2013) realizaram um estudo com crianças do $4^{\circ}$ ao $8^{\circ}$ ano do Ensino Fundamental, a fim de comparar e caracterizar o desempenho de escolares com TDAH e sem queixas de aprendizagem em tarefas metalinguísticas e de leitura. Foram encontradas diferenças estatisticamente significativas entre os grupos nas tarefas de manipulação silábica e fonêmica, bem como nas provas de leitura de palavras reais regulares e irregulares e pseudopalavras. Nesse estudo, concluiu-se que as crianças com TDAH apresentaram desempenho inferior nas tarefas de CF consideradas mais complexas, como a manipulação de sílabas e fonemas. Além disso, eles também apresentaram prejuízos na leitura de palavras irregulares, isto é, palavras que não obedecem às regras de correspondência letra-som da língua e cuja pronúncia deve ser memorizada.

A memória de trabalho fonológica (MTF) está envolvida no processamento temporário e na estocagem de informações, sendo caracterizada por ter capacidade limitada e ser encarregada de armazenar brevemente as informações em um código fonológico (Baddeley \& Hitch,1974). A capacidade de memória de trabalho é determinante para a compreensão de leitura (Seigneuric \& Ehrlich, 2005; Swanson, 1999), pois é necessário memorizar os segmentos sonoros lidos para atribuir sentido a uma frase, armazenar o significado de cada uma, até entender um parágrafo e guardar o significado dos parágrafos para alcançar o entendimento geral do texto. Estudos (Cain et al., 2004; Oakhill et al., 1998; Savage et al., 2007) indicam uma relação entre deficits na MTF e baixo desempenho em leitura. De acordo com Van der Leij e Morfidi (2006), escolares com dificuldades na leitura apresentam fluência alterada e problemas com a compreensão de leitura em decorrência de alterações no processamento fonológico, o 
qual também envolve a baixa capacidade de armazenamento e de recuperação de informação fonológica na MT. Além disso, pesquisas (Bolden et al., 2012; Raiker et al., 2017) também indicam que crianças com diagnóstico de TDAH apresentam desempenho aquém em tarefas de MTF quando comparadas a um grupo sem o diagnóstico. No entanto, é importante ressaltar que Friedman et al. (2017), em um estudo que investigou o papel de três componentes da Memória de Trabalho (Executivo Central, Alça Fonológica e Esboço Visuoespacial) na leitura em crianças com TDAH, observaram que apenas o Executivo Central mediou a relação entre o TDAH e a compreensão de leitura. Então, ainda não é clara a relação da MTF com a leitura em crianças com TDAH.

A Nomeação Seriada Rápida (NSR) ou Nomeação Automatizada Rápida (RAN) é indexada pela velocidade para acessar e recuperar rótulos verbais na nomeação contínua de estímulos visuais dispostos em séries (Denckla \& Rudel, 1976). De acordo com Justi (2015), a natureza da nomeação seriada rápida ainda não é evidente, sendo que alguns autores a associam a recuperação de códigos fonológicos na memória de longo prazo (Wagner \& Torgensen, 1987), velocidade de processamento geral (Kail \& Hall, 1994), falhas nos processos que dão suporte à abstração de padrões ortográficos (Bowers, 2001), ou problemas na sincronização e automatização de diversos processos de natureza perceptual, lexical e motora (Wolf \& Bowers, 1999). A despeito disso, existem vários estudos que indicam que há relação da NSR com a leitura, mesmo quando diferentes variáveis são controladas (Justi \& Roazzi, 2012; Mousinho, 2003; Wolf \& Bowers, 1999).

No que se refere aos indivíduos com TDAH, estudos (Capellini et al., 2007; Semrud-Clikeman et al., 2000) também indicam que estes podem apresentar prejuízo na NSR. Capellini et al. (2007) investigaram o desempenho na NSR de escolares brasileiros na faixa etária de 8 a 12 anos. Eles foram divididos em três grupos: grupo com TDAH (GTDAH), grupo com dislexia e grupo controle (GC). Os resultados evidenciaram que tanto o GTDAH quanto o grupo com dislexia apresentaram comprometimento na NSR em relação ao GC, indicando que a NSR pode ser considerada importante para o desempenho na leitura.

Como foi possível observar, a leitura é uma habilidade que envolve diferentes aspectos, como precisão, fluência e compreensão. Além disso, há um conjunto variado de processos cognitivos e habilidades metalinguísticas que são importantes para o aprendizado da leitura. Dada a relação entre o TDAH e o baixo desempenho acadêmico (DuPaul \& Stoner, 2007) e a importância da leitura para o último, é importante compreender melhor a relação entre o TDAH e a leitura. Desse modo, o presente estudo investigou a relação do TDAH com a leitura considerando os diferentes aspectos da última (precisão, fluência e compreensão), bem como os diferentes processos cognitivos e habilidades metalinguísticas que são importantes para a leitura (CF, MTF, NSR, atenção, vocabulário e flexibilidade cognitiva).

\section{MÉTODO}

\section{Participantes}

Participaram deste estudo alunos matriculados em 19 escolas de uma cidade de médio porte do interior do estado de Minas Gerais. As escolas foram escolhidas a partir da existência de crianças com diagnóstico de TDAH. Só foram incluídas na pesquisa aquelas diagnosticadas com TDAH, que possuíam laudo médico atestando o diagnóstico e que não obtiveram escore zero em todas as tarefas de leitura (vide descrição das tarefas na seção 'instrumentos'). A aplicação desse critério resultou em 35 crianças diagnosticadas com TDAH e 35 crianças que compuseram o grupo sem TDAH (vide seção 'procedimentos' para informações sobre o pareamento); desse total, 26 do sexo feminino (37\%) e 44 do sexo masculino (63\%). A idade média dos participantes da pesquisa foi de 125,66 meses $(D P=18,66)$. Das 70 crianças participantes, 6 eram do $2^{\circ}$ ano ( 4 meninos), 10 do $3^{\circ}$ ano ( 7 meninos), 16 do $4^{\circ}$ ano ( 10 meninos), 16 do $5^{\circ}$ ano ( 7 meninos), 16 do $6^{\circ}$ ano ( 10 meninos) e 6 do $7^{\circ}$ ano (6 meninos). Sessenta cursavam o EF em escolas públicas e 10 em escolas particulares. Dentre as 35 crianças com o transtorno, 16 faziam uso de metilfenidato (Ritalina $\left.{ }^{\circledR}\right)$ e 13 apresentavam TDAH com algum tipo de comorbidade.
Não foram incluídas na amostra crianças com algum tipo de deficiência sensorial e crianças não alfabetizadas.

\section{Instrumentos}

Escala de sintomas para TDAH. Foi utilizada a escala SNAP- IV (Mattos et al., 2006). Nessa escala, os critérios do DSM-TR-IV (American Psychiatric Association, 2000) para diagnóstico de TDAH foram incluídos em subconjuntos de sintomas, sendo os itens de 1-9 referentes à desatenção e os itens de 10-18 referentes à hiperatividade e impulsividade. $\mathrm{O}$ instrumento foi preenchido, individualmente, pelos professores de Língua Portuguesa e pelos responsáveis pelas crianças.

Consciência fonológica. Foi aplicada a tarefa de Spoonerismo, retirada do trabalho de Justi e Roazzi (2012), que requer que o participante troque o primeiro som de duas palavras apresentadas um pelo outro (Ex.: /miLU veRdi/=/ vilLU meRdi/). Em relação ao escore, a criança não recebe pontos quando erra as duas palavras, recebe um ponto quando acerta apenas uma palavra e recebe dois pontos quando responde corretamente as duas palavras. 
Memória de Trabalho Fonológica. Para avaliar a memória de trabalho fonológica, foi utilizado o subteste 'dígitos' da terceira edição da Escala de Inteligência Weschsler para crianças, o WISC-III (Wechsler, 2002). Este subteste é formado por dois conjuntos: Ordem Direta e Ordem Inversa. Na ordem direta, a criança deve repetir uma sequência de dígitos na mesma ordem enunciada pelo examinador. Já na ordem inversa, a criança deve repetir uma sequência de dígitos na ordem inversa daquela enunciada pelo examinador. O subteste foi administrado e corrigido de acordo com as especificações do manual.

Medidas de Nomeação Seriada Rápida. Na tarefa de Nomeação Seriada Rápida, o participante nomeia, da esquerda para direita, tão rápido e corretamente quanto possível, um conjunto de 50 estímulos visuais, todos de alta frequência de ocorrência, dispostos em série em um cartão (Justi \& Roazzi, 2012). Os estímulos visuais a serem nomeados são: quadrados coloridos - na tarefa de nomeação seriada rápida de cores; números - na tarefa de nomeação seriada rápida de números; objetos desenhados - na tarefa de nomeação seriada rápida de objetos; e letras - na tarefa de nomeação seriada rápida de letras (Justi et al., 2014). $\mathrm{O}$ escore consistiu no número de estímulos pronunciados corretamente dividido pelo tempo total gasto, em segundos, para a leitura dos estímulos.

Fluência de leitura. Foi utilizado o Teste de Fluência de Leitura - TFL (Justi \& Roazzi, 2012) que consiste em um cartão com 60 palavras que devem ser lidas da esquerda para direita, em voz alta, o mais rápido possível. O escore consiste no número de palavras lidas corretamente em um tempo de 30 segundos.

Precisão de leitura. Foi aplicado o subteste de leitura do Teste de Desempenho Escolar - TDE (Stein,1994). O participante deve ler palavras isoladas que variam em termos de frequência, regularidade e extensão. $\mathrm{O}$ teste foi corrigido conforme as sugestões de Lúcio e Pinheiro (2014) e o escore consistiu no número de palavras lidas corretamente.

Compreensão de texto. O teste Cloze foi utilizado para medir a compreensão de leitura (Santos et al., 2002). O teste consiste em um texto, do qual omite-se o quinto vocábulo e os participantes devem preencher a lacuna com a palavra que completa melhor o sentido do texto. Os escores são obtidos somando-se os números de lacunas preenchidas corretamente. Para crianças até $\mathrm{o} 5^{\circ}$ ano, foi aplicado o texto "Uma vingança infeliz" (Cloze A) e para crianças do $6^{\circ} \mathrm{e}$ $7^{\circ}$ ano, foi utilizado o texto "Coisas da natureza" (Cloze B), ambos desenvolvidos por Santos (2004). Foi utilizada a correção literal, que preconiza como acerto o preenchimento da palavra exata que foi omitida.

Medida de Atenção. Foi utilizado o Teste de Atenção por Cancelamento (Montiel \& Seabra, 2012), que avalia a atenção seletiva e consiste em três matrizes impressas com diferentes tipos de estímulos e níveis de dificuldade. O participante deve assinalar todos os estímulos iguais ao estímulo alvo previamente determinado. Na correção, considera-se o número de estímulos assinalados corretamente em um tempo de 60 segundos. A pontuação foi computada conforme as normas do manual.

Medidas de atenção e flexibilidade cognitiva. Foi utilizado o Teste de Trilhas (TT) (Montiel \& Seabra, 2012) para avaliar, especificamente, a flexibilidade cognitiva, além da atenção visual sustentada. O teste é dividido em duas partes: a parte "A" é referida à avaliação da atenção sustentada visual, sendo empregada na grande maioria das vezes como controle ou linha de base para a interpretação do desempenho na parte "B", que caracteriza uma medida de atenção, velocidade e, sobretudo, flexibilidade cognitiva. O participante tem 60 segundos para concluir cada parte da tarefa (A e B). A parte "A" do teste é composta por letras e números, em duas etapas. Na primeira etapa, o participante deve ligar as letras de A até $\mathrm{L}$ em ordem alfabética e, na segunda, deve ligar os números de 1 até 12, em ordem crescente. Na parte "B" há 12 letras, de A até $\mathrm{L}$, e 12 números, de 1 até 12 . O participante deve ligar letras e números alternadamente, iniciando na letra "A" e finalizando no número " 12 ". Para a correção, foi considerada a pontuação normatizada, conforme o manual.

Medidas de inteligência. Foram utilizados os testes Vocabulário e Cubos da Escala de Inteligência Wechsler para crianças - WISC III (Wechsler, 2002). O teste Vocabulário compõe-se de uma série de palavras, que são apresentadas oralmente pelo examinador, cujas definições devem ser fornecidas oralmente pelas crianças. Já no teste Cubos o participante utiliza cubos coloridos para reproduzir até 13 figuras de duas cores, sendo que há um tempo para cada montagem, marcado no cronômetro (Wechsler, 2002). Os testes foram aplicados e corrigidos conforme as instruções do manual. Para avaliar a inteligência, utilizou-se o somatório dos escores nos subtestes Cubos e Vocabulário para o cálculo do QI Estimado, conforme sugerido por Mello et al. (2011).

\section{Procedimentos}

Após a aprovação da pesquisa pelo Comitê de Ética de uma Universidade Federal, parecer número 1.431.853, e do consentimento das secretarias Municipal e Estadual de Educação, iniciou-se o recrutamento de crianças diagnosticadas com TDAH nos Centros de Atendimento Educacional Especializado da prefeitura (CAEEs) e posteriormente nas escolas públicas e privadas. Após o consentimento dos responsáveis legais pelas crianças e da direção das escolas, a coleta de dados ocorreu na escola dos participantes em dias, horários e local previamente estipulados pela direção.

Destaca-se que, para a realização da pesquisa, foram compostos dois grupos de crianças: um com TDAH (GTDAH) e outro sem (grupo controle/GC). Para a composição do GTDAH, as crianças deveriam possuir o transtorno, informação comprovada por meio de laudo médico. O diagnóstico e a idade (7 a 13 anos incompletos), 
bem como a matrícula no ensino regular, foram requisitos para a composição do grupo. Para a composição do GC, houve a colaboração dos professores de Língua Portuguesa, que indicaram uma criança da mesma sala da criança com TDAH, que deveria apresentar rendimento acadêmico mediano e ter idade próxima à da criança com TDAH. Este procedimento foi utilizado para controlar a idade, o nível educacional e o ambiente educacional entre os grupos. A aplicação das tarefas ocorreu individualmente e variou de duas a quatro sessões.

\section{RESULTADOS}

Com exceção dos escores referentes à escala SNAPIV que foram assumidos como ordinais devido às suas naturezas, optou-se por avaliar se os demais dados apresentaram distribuição normal. Para isso, utilizou-se o teste Kolmogorov-Smirnov, porque permite comparar a qualidade do ajuste (goodness-of-fit) dos dados obtidos em relação a uma distribuição hipotética de interesse (Arnold \& Emerson, 2011) que, no caso do presente estudo, é a distribuição normal. Nesse caso, valores alfa menores do que 0,05 são aceitos como critério para rejeitar a hipótese de que a distribuição dos dados se assemelha a uma distribuição normal. Os resultados dos testes de Kolmogorov-Smirnov indicaram que, com exceção dos testes TT partes A e B $(p<0,001$ e $p<0,05$, respectivamente), TAC parte 1 ( $p<$ $0,01)$ e vocabulário $(p=0,08$ que ficou próximo do critério para se rejeitar a normalidade), todas as outras variáveis apresentaram distribuição normal (todos os $p>0,13$ ). Salienta-se que, para verificar se os escores obtidos no teste
Cloze apresentaram distribuição normal, realizou-se uma análise separada para os dois tipos deste teste. Cloze A: "Uma vingança infeliz", aplicado em alunos do $2^{\circ}$ ao $5^{\circ}$ anos $(n=48)$ e Cloze B: "Coisas da natureza", aplicado em alunos do $6^{\circ}$ e $7^{\circ}$ anos $(n=22)$. Ambos apresentaram distribuição normal, com $p=0,20$ e $p=0,44$, respectivamente. As estatísticas descritivas podem ser observadas na Tabela 1.

Como esperado, houve alta correlação entre as medidas de NSR $(r=0,71$ a $r=0,88)$ e entre os escores totais da escala SNAP-IV preenchida pela família e pelos professores $(\rho=0,704)$. Assim sendo, optou-se por utilizar nas análises o escore médio das quatro medidas de Nomeação Seriada Rápida (objetos, cores, números e letras) e o escore total da escala SNAP-IV preenchida pelos professores, pois duas famílias deixaram de preencher a escala.

Ressalta-se que, das 35 crianças diagnosticadas com TDAH, 16 faziam uso de (Metilfenidato) e 19 não. Então, para verificar se as crianças com TDAH compõem um grupo

Tabela 1

Estatísticas Descritivas

\begin{tabular}{|c|c|c|c|c|c|c|c|}
\hline Variável & $N$ & Máx. O & Mín. O & Média & D.P. & Assimetria & Curtose \\
\hline Idade & 70 & 154 & 85 & 125,66 & 18,66 & $-0,33$ & $-0,81$ \\
\hline TDE & 70 & 25 & 0 & 9,98 & 7,58 & 0,28 & $-1,12$ \\
\hline TFL & 70 & 60 & 2 & 26,71 & 16,88 & 0,49 & $-0,87$ \\
\hline Cloze A & 48 & 11 & 0 & 3,85 & 2,95 & 0,74 & 0,05 \\
\hline Cloze B & 22 & 28 & 3 & 20,95 & 6,07 & $-1,32$ & 2,25 \\
\hline NSR objetos & 70 & 1,92 & 0,58 & 1,16 & 0,27 & 0,08 & 0,12 \\
\hline NSR cores & 70 & 1,79 & 0,38 & 1,04 & 0,30 & 0,20 & $-0,32$ \\
\hline NSR números & 70 & 3,57 & 0,55 & 1,69 & 0,53 & 0,67 & 1,41 \\
\hline NSR letras & 70 & 2,94 & 0,78 & 1,64 & 0,49 & 0,62 & 0,08 \\
\hline Dígitos & 70 & 16 & 3 & 8,87 & 2,77 & 0,34 & $-0,26$ \\
\hline Spooneirismo & 70 & 20 & 0 & 12,03 & 6,30 & $-0,55$ & $-0,85$ \\
\hline Vocabulário & 70 & 18 & 5 & 11,58 & 3,31 & $-0,17$ & $-0,67$ \\
\hline QI estimado & 70 & 138 & 68 & 104,66 & 16,83 & $-0,18$ & $-0,56$ \\
\hline TT A & 70 & 111 & 0 & 75,17 & 46,15 & $-0,86$ & $-1,14$ \\
\hline TT B & 70 & 141 & 0 & 93,20 & 32,83 & $-1,37$ & 1,74 \\
\hline TAC 1 & 70 & 120 & 66 & 105,54 & 11,44 & $-1,38$ & 1,65 \\
\hline TAC 2 & 70 & 139 & 0 & 98,33 & 19,19 & $-1,77$ & 9,09 \\
\hline TAC 3 & 70 & 151 & 52 & 105,17 & 17,84 & $-0,26$ & 1,34 \\
\hline
\end{tabular}

Nota . Máx. $\mathrm{O}=$ Escore Máximo Obtido; $\mathrm{M} . \mathrm{O}=$ Escore Mínimo Obtido; D.P.= Desvio Padrão; TFL = Teste de Fluência de Leitura; NSR = Nomeação Seriada Rápida; TDE = Teste de Desempenho Escolar; TT A = Teste de Trilhas parte A; TT B = Teste de Trilhas parte B; TAC $1=$ Teste de Atenção por Cancelamento parte 1; TAC 2 = Teste de Atenção por Cancelamento parte 2; TAC 3 = Teste de Atenção por Cancelamento parte 3; Cloze A= aplicado a crianças do $2^{\circ}$ ao $5^{\circ}$ ano do Ensino Fundamental; Cloze $\mathrm{B}=$ aplicado a crianças do $6^{\circ}$ ao $7^{\circ}$ ano do Ensino Fundamental. 
homogêneo em relação ao seu desempenho nas tarefas aplicadas, foram realizadas separadamente dois tipos de comparação: uma entre crianças diagnosticadas com TDAH, que faziam uso de medicação e seus respectivos controles ( $n$ $=32$ ) e outra entre crianças diagnosticadas com o transtorno, que não faziam uso de medicação e seus respectivos controles $(n=38)$. Devido ao tamanho da amostra ser reduzido, optou-se pelo uso do teste não paramétrico de Mann-Whitney U. Não foram realizadas as análises de comparação para o teste Cloze $\mathrm{B}$ relativo às crianças do $5^{\circ}$ e $6^{\circ}$ anos, porque a amostra ficou muito pequena.

A partir da aplicação do teste de Mann-Whitney U, constatou-se que os grupos diagnosticados com TDAH (medicados e não medicados) diferiram dos seus respectivos controles nos três tipos de escore da escala SNAP-IV, sendo todos os $p \leq 0,001$. Além dos escores da SNAP-IV, as crianças diagnosticadas com TDAH que faziam uso de medicação diferiram de forma estatisticamente significativa de seus respectivos controles apenas no teste TT parte $\mathrm{A}(\mathrm{U}=$ $47,5 ; z=-3,13 ; p<0,01)$. Já as crianças diagnosticadas com TDAH que não faziam uso de medicação diferiram de seus respectivos controles no TDE, TFL, Cloze A, NSR, CF, MTF e TAC parte 2 (todos com $p<0,05$ ), sendo que, para o TT parte A e para o QI, o nível alpha ficou próximo do esperado ( $p=0,06$ e $p=0,07$, respectivamente). Basicamente, $\mathrm{o}$ GTDAH que não fazia uso de medicação só não diferiu de seus respectivos controles no teste de vocabulário $(p=$ $0,14)$, no TT parte B $(p=0,13)$ e no TAC partes 1 e $3(p=$ 0,$58 ; p=0,50$, respectivamente). Destarte, optou-se por não tratar o GTDAH como homogêneo e foi criada uma nova variável ordinal para considerar a "severidade dos sintomas de TDAH", com os valores: $0=$ crianças sem diagnóstico de TDAH; 1 = crianças com diagnóstico de TDAH, que fazem uso de medicação; e 2 = crianças com diagnóstico de TDAH, que não fazem uso de medicação. Essa variável foi utilizada para avaliar a relação do TDAH com a leitura em todas as análises subsequentes. Por fim, é necessário pontuar que não foi possível controlar as comorbidades neste estudo, devido à sua heterogeneidade.

\section{Análises TDAH e leitura}

Para avaliar a relação entre TDAH e leitura (precisão, fluência e compreensão), foram realizadas, preliminarmente, diversas análises de regressão linear múltipla, tendo como variável critério: ora o TDE, ora o TFL e ora o Cloze A. O Cloze B não foi incluído nas análises de regressão, por se tratar de uma amostra pequena $(n=22)$. O objetivo foi mapear as variáveis que apresentavam relação com a leitura e que contribuíam para essa habilidade com variância adicional à do TDAH. Em todas as análises realizadas, a idade e a inteligência foram controladas. As análises para o TDE e o TFL envolveram três passos: a idade cronológica e a inteligência entravam no primeiro passo; no segundo, a variável de grupo "severidade dos sintomas de TDAH"; e, no terceiro, apenas uma das variáveis potencialmente explicativas da leitura (CF ou Vocabulário ou TT, etc.). Já as análises para o Cloze A envolveram apenas dois passos. No primeiro passo, entraram idade cronológica e inteligência; no segundo passo entraram, separadamente, cada uma das seguintes variáveis: "severidade dos sintomas de TDAH" e "variáveis potencialmente explicativas da leitura" (CF ou Vocabulário ou TT, etc.).

Após a identificação das variáveis que se relacionavam com a leitura, foi realizada uma nova análise de regressão, incluindo todas as variáveis potencialmente explicativas da leitura com o intuito de verificar quais delas contribuíam mais fortemente para a precisão, fluência e compreensão de leitura. Ressalta-se que, por questões de espaço, no que diz respeito às análises preliminares, será informado aqui apenas as variáveis que apresentaram contribuições significativas (as tabelas referentes às análises preliminares podem ser obtidas como material suplementar). A análise que incluiu as principais variáveis explicativas será apresentada em detalhes.

\section{TDAH e precisão de leitura $(N=70)$}

Nas análises preliminares, entrando após as variáveis 'idade' e 'inteligência', a variável "severidade dos sintomas de TDAH" explicou apenas 2,3\% de variância adicional na precisão de leitura medida pelo TDE, sendo que essa mudança em $R^{2}$ não chegou a ser estatisticamente significativa $(p=0,08)$. Ao entrar no terceiro passo, as únicas variáveis que explicaram variância adicional na precisão de leitura de forma estatisticamente significativa foram: NSR (mudança em $R^{2}$ de $12,9 \% ; p<0,001$ ); CF (mudança em $R^{2}$ de $12,7 \% ; p<0,001$ ); MTF (mudança em $R^{2}$ de $9,4 \% ; p<0,001$ ) e TT parte A (mudança em $R^{2}$ de $3,8 \% ; p=0,022)$. Nenhuma das outras variáveis incluídas no terceiro passo da análise (Vocabulário, TT parte B e TAC partes 1, 2 e 3) explicaram variância adicional de forma estatisticamente significativa (para todas as mudanças em $\left.R^{2}, p>0,06\right)$.

Como os resultados das análises preliminares indicaram que a NSR, a CF, a MTF e o TT parte A explicaram variância adicional em relação a idade, a inteligência e a severidade dos sintomas de TDAH, realizou-se então uma nova análise de regressão linear, considerando em conjunto a contribuição dessas quatro variáveis para a precisão de leitura medida pelo TDE. Essa análise foi realizada incluindo todas as quatro variáveis em um único passo. Os resultados da análise de regressão indicaram que, em conjunto, a NSR, a CF, a MTF e o TT parte A foram responsáveis por $68,4 \%$ da variância no TDE. No entanto, apenas os coeficientes de regressão da NSR e da CF foram estatisticamente significativos $(t=5,88, p<0,001$ e $t=3,25$, $p<0,01$, respectivamente). Ou seja, ao serem tomados em 
conjunto com a NSR e a CF, a contribuição da MTF e do TT parte A deixaram de ser estatisticamente significativas (todos os $p>0,25$ ). Por fim, os coeficientes de regressão padronizados indicaram uma contribuição mais forte da NSR do que da CF ( $\beta=0,52$ e $\beta=0,33$, respectivamente). Uma simulação dos resultados dessa análise, utilizando-se o método de bootstrapping com mil amostras, apresentou os mesmos resultados. Isso é, todos os coeficientes de regressão que eram estatisticamente significativos continuaram sendo e todos os que não eram estatisticamente significativos assim permaneceram.

\section{TDAH e fluência de leitura $(n=70)$}

Nas análises preliminares, entrando após as variáveis 'idade' e 'inteligência', a variável "severidade dos sintomas de TDAH" explicou apenas $1,3 \%$ de variância adicional na fluência de leitura medida pelo TFL, sendo que essa mudança em $R^{2}$ não foi estatisticamente significativa ( $p=$ 0,23 ). Ao entrar no terceiro passo, as únicas variáveis que explicaram variância adicional na fluência de leitura de forma estatisticamente significativa foram: NSR (mudança em $R^{2}$ de 27\%; $p<0,001$ ); CF (mudança em $\mathrm{R}^{2}$ de $19,1 \%$; $p<0,001$ ); MTF (mudança em $R^{2}$ de $9,7 \%$; $p<0,001$ ); Vocabulário (mudança em $R^{2}$ de $5,4 \% ; p=0,01$ ); e TAC parte 3 (mudança em $R^{2}$ de $7,1 \% ; p<0,01$ ). Nenhuma das outras variáveis incluídas no terceiro passo da análise (TT partes A e B e TAC partes 1 e 2) explicaram variância adicional de forma estatisticamente significativa (para todas as mudanças em $R^{2}, p>0,11$ ).

Como os resultados das análises preliminares indicaram que a NSR, a CF, a MTF, o Vocabulário e o TAC parte 3 explicaram variância adicional em relação a idade, a inteligência e a severidade dos sintomas de TDAH, realizouse então uma nova análise de regressão linear, considerando, em conjunto, a contribuição dessas cinco variáveis para a fluência de leitura medida pelo TFL. A análise foi realizada incluindo-se todas as cinco variáveis em um único passo. Os resultados da análise de regressão indicaram que, em conjunto, a NSR, a CF, a MTF, o Vocabulário e o TAC parte 3 foram responsáveis por $79 \%$ da variância no TFL. No entanto, apenas os coeficientes de regressão da NSR e da $\mathrm{CF}$ foram estatisticamente significativos $(t=7,73 ; p<0,001$ e $t=3,60 ; p=0,001$, respectivamente). Ou seja, ao serem tomados em conjunto com a NSR e a $\mathrm{CF}$, a contribuição da MTF, do Vocabulário e do TAC parte 3 deixaram de ser estatisticamente significativas (todos os $p>0,35$ ). Por fim, os coeficientes de regressão padronizados indicaram uma contribuição mais forte da NSR do que da CF $(\beta=0,63 \mathrm{e}$ $\beta=0,30$, respectivamente). Uma simulação dos resultados dessa análise, utilizando-se o método de bootstrapping com mil amostras, apresentou os mesmos resultados.

\section{TDAH e compreensão de leitura $(n=48)$}

Os resultados a seguir consideram apenas os dados das análises referentes ao teste Cloze $\mathrm{A}$, que foi aplicado do $2^{\circ}$ ao $5^{\circ}$ ano (não houve tamanho amostral suficiente para analisar o Cloze B que foi aplicado no $6^{\circ}$ e $7^{\circ}$ anos). Essas análises contaram com 48 crianças, portanto, optou-se por limitar as análises preliminares a três variáveis para atender o critério de Dancey e Reidy (2006) de, pelo menos, 15 participantes por variável nas análises de regressão. Nessas análises preliminares, a 'idade' e a 'inteligência' entravam no primeiro passo e uma das variáveis preditivas entrava no segundo.

Nas análises preliminares, entrando após as variáveis 'idade' e 'inteligência', a variável "severidade dos sintomas de TDAH" explicou $25,7 \%$ de variância adicional na compreensão de leitura medida pelo Cloze A, sendo essa mudança em $R^{2}$ estatisticamente significativa $(p<0,001)$. Além disso, ao entrar no segundo passo, as únicas variáveis que explicaram variância adicional na compreensão de leitura de forma estatisticamente significativa foram: NSR (mudança em $r^{2}$ de 16,8\%; $p=0,001$ ); CF (mudança em $r^{2}$ de $30,3 \% ; p<0,001$ ) e MTF (mudança em $r^{2}$ de 26,2\%; $p<$ $0,001)$. Nenhuma das outras variáveis incluídas no segundo passo da análise (Vocabulário, TT partes A e B, e TAC partes 1, 2 e 3) explicaram variância adicional de forma estatisticamente significativa (para todas as mudanças em $\left.R^{2}, p>0,13\right)$.

Como nas análises anteriores a MTF sempre deixou de contribuir quando considerada em conjunto com a CF e a NSR, optou-se por realizar mais uma análise preliminar. Nessa análise, as variáveis NSR, CF e MTF foram incluídas em um único passo. Os resultados indicaram que apenas os coeficientes de regressão da NSR e da CF foram estatisticamente significativos $(t=3,22 ; p=0,002$ e $t=$ 2,$79 ; p=0,008$, respectivamente). Ou seja, ao ser incluída em conjunto com a NSR e a CF, a contribuição da MTF deixou de ser estatisticamente significativa $(p=0,085)$. Por fim, realizou-se uma análise final, na qual as variáveis "severidade dos sintomas de TDAH", CF e NSR, entraram juntas em um único passo. Os resultados da análise de regressão indicaram que, em conjunto, a "severidade dos sintomas de TDAH", a CF e a NSR, foram responsáveis por $66 \%$ da variância no Cloze A. Todos os coeficientes de regressão foram estatisticamente significativos (todos $p<$ 0,05 ) e os coeficientes de regressão padronizados indicaram que a contribuição mais forte foi da $\mathrm{CF}$, sendo seguida da contribuição da "severidade dos sintomas de TDAH" e da contribuição da NSR $(\beta=0,46 ; \beta=-0,38$; e $\beta=0,22$, respectivamente). Uma simulação dos resultados dessa análise, utilizando o método de bootstrapping com mil amostras, apresentou os mesmos resultados. 


\section{DISCUSSÃO}

Para avaliar se as crianças diagnosticadas com TDAH diferiam das crianças do grupo controle nos sintomas associados ao transtorno, os dois grupos foram comparados em seus escores obtidos na escala SNAP-IV (professores) e os resultados dessa análise indicaram que os grupos diferiram nesses escores. Além disso, as crianças com TDAH medicadas em comparação com seus pares apresentaram um desempenho melhor do que as crianças com TDAH não medicadas em comparação com seus pares. Assim sendo, em conjunto, esses dados servem como corroboração dos laudos diagnósticos de TDAH, nos quais a seleção dos participantes da presente pesquisa foi baseada.

Como a amostra deste estudo foi composta por crianças com TDAH medicadas e não medicadas, optou-se por incluir o fator 'uso de medicação' como parte da variável 'severidade dos sintomas de TDAH'. As crianças com TDAH e sem medicação representavam a condição mais severa nos sintomas do transtorno. Isto é um diferencial desse estudo, já que, em geral, estudos no Brasil que investigam a leitura em crianças com TDAH não controlam o uso da medicação, como, por exemplo, os estudos de Lobo e Lima (2008) e de Silva et al. (2011). Controlar a medicação é importante, afinal, de acordo com Greenhill et al. (1999), cerca de 70\% dos indivíduos com TDAH que fazem uso do metilfenidato apresentam melhoras no desempenho escolar.

Nas tarefas de leitura, pôde-se perceber que, após controlar a idade e a inteligência, a severidade dos sintomas de TDAH só contribuiu de forma estatisticamente significativa para explicar variação na compreensão de leitura. Tal relação é esperada à luz de outros estudos, como o de Fergusson e Horwood (1992), no qual a principal medida de leitura indexava a compreensão. $\mathrm{O}$ estudo de Oliveira et al. (2011) realizado no Brasil também atesta a relação do TDAH com a compreensão de leitura, indicando que crianças com o transtorno apresentam pior desempenho na compreensão de leitura em relação ao GC. Além disso, no presente estudo, o TDAH continuou contribuindo para explicar variação na compreensão de leitura, mesmo após a inclusão da CF e da NSR na equação regressiva. Este dado é importante, pois é um indicativo de que o prejuízo na compreensão de leitura das crianças com TDAH não decorre de um deficit nessas variáveis. Outro estudo que apresenta resultados similares aos do presente estudo é o de Friedman et al. (2017). Eles observaram que, mesmo após considerar a habilidade de conversão ortográfica (medida que incluía a precisão e a fluência de leitura combinadas) como mediadora da relação do TDAH com a compreensão de leitura, o diagnóstico de TDAH continuou explicando significativamente essa variável. Esse resultado indica que a relação do TDAH com a compreensão de leitura não se deve primordialmente à fluência ou precisão de leitura e é compatível com o achado do presente estudo, no qual o
TDAH não contribuiu para explicar a precisão e a fluência de leitura, após o controle da idade e da inteligência.

O prejuízo que a maioria das crianças com TDAH apresenta na função executiva (Sesma et al., 2009) é uma possível explicação para a especificidade da relação entre o TDAH e a compreensão de leitura observada no presente estudo. Segundo Ghelani et al. (2004), crianças com TDAH que não apresentam prejuízo na precisão de leitura ainda podem ter problemas quanto à compreensão textual. Nessa direção, o estudo de Friedman et al. (2017) demonstrou que foram os aspectos executivos relacionados à memória de trabalho que apresentaram o papel mais relevante na relação do diagnóstico de TDAH com a compreensão de leitura. Além disso, outros estudos indicam que a função executiva se relaciona com a compreensão de leitura mesmo em pessoas sem diagnóstico de TDAH (Cutting et al., 2009; Georgiou \& Das, 2016; Potocki et al., 2015). Segundo Cutting et al. (2009), estudantes com dificuldades específicas de compreensão de leitura mostram desempenho significativamente inferior em testes de função executiva, quando comparados com leitores proficientes. Em especial, o estudo de Potocki et al. (2015), apontou forte poder preditivo da função executiva na compreensão de textos para questões inferenciais, mesmo sendo controlados preditores linguísticos da compreensão, como a decodificação de palavras, o vocabulário e as habilidades sintáticas. É interessante notar que os componentes da função executiva avaliados por Potocki et al. (2015) foram importantes para a compreensão de textos, mas não para a precisão e fluência de leitura. Este é o mesmo padrão que, no presente estudo, as crianças com TDAH apresentaram; isto é, a severidade dos sintomas de TDAH não influiu na precisão e na fluência, mas sim na compreensão. Tal resultado condiz com a hipótese de que a relação entre TDAH e compreensão de leitura seja decorrente do deficit na função executiva que as crianças com TDAH em geral possuem.

A relação entre função executiva e o teste Cloze utilizado no presente estudo torna-se intuitiva se considerarmos que os componentes dessa função (monitoramento e planejamento) são exigidos para um bom desempenho no teste. Afinal, uma das instruções é que a pessoa tente ler todo o texto primeiro, para só depois tentar preencher as lacunas. Assim, o bom desempenho no Cloze demanda planejamento estratégico, organização e inibição de resposta para evitar que o preenchimento das lacunas ocorra com a primeira palavra que vem à mente. Nesse sentido, o estudo de Arrington et al. (2014) enfatiza que o planejamento está significativamente relacionado à compreensão de leitura, assim como a velocidade de processamento e de nomeação, a fluência e a memória operacional. Além disso, Bovo et al. (2016) argumentam que o controle inibitório, responsável pela diminuição das interferências na atenção e no pensamento, 
também é importante para a compreensão de leitura. Por fim, Oakhill et al. (2005) resumem o papel da função executiva na leitura ao afirmarem que o leitor deve utilizar estratégias para monitorar a compreensão, identificar incongruências e realizar ajustes durante a intepretação de um texto.

Ainda no que diz respeito à compreensão de leitura, é importante considerar que, além do TDAH, a CF e a NSR também contribuem para essa habilidade. Esse resultado não é surpreendente, visto que, nas últimas décadas, os pesquisadores descobriram que o sucesso ou o fracasso na compreensão de leitura é, em grande parte, determinado pela capacidade de decodificação ou leitura de palavras isoladas (Adams, 1990; Lyon, 1995; Torgensen, 2000). Para aprender a decodificar, a criança deve ser capaz de manipular a estrutura sonora da fala e entender que as palavras são compostas por fonemas. As crianças com dificuldades na decodificação apresentam deficit específicos no processamento fonológico (Lyon, 1995), o que afeta negativamente a compreensão de leitura. A falta de fluência também impede a compreensão, mesmo quando a precisão de leitura é alcançada, porque a leitura passa a requerer um esforço cognitivo sustentado por um período de tempo maior (Perfetti \& Hogaboam, 1975; Perfetti et al., 1996). Como há boas evidências de que a CF e a NSR contribuem para a precisão e a fluência da leitura (Justi \& Roazzi, 2012) e como elas foram as principais variáveis explicativas da precisão e da fluência de leitura no presente estudo, não surpreende que também tenham apresentado uma relação significativa com a compreensão de leitura, conforme já observado em outros estudos (Correa \& Mousinho, 2013; Mousinho, et al., 2009; Salles, 2009).

Em suma, é possível que o TDAH acarrete pior compreensão de leitura em razão da ocorrência de prejuízo na função executiva, enquanto a consciência fonológica e a nomeação seriada rápida se relacionem com a compreensão de leitura, principalmente via sua contribuição para a precisão e a fluência de leitura. Esta é uma possibilidade interessante, pois sinaliza que a contribuição dessas três variáveis para a compreensão de leitura é de natureza diferente. Para testar essa hipótese, realizou-se uma análise post hoc de regressão linear múltipla, na qual entraram no primeiro passo as medidas de precisão (TDE) e fluência de leitura (TFL) e no segundo passo entraram, alternadamente, a CF, a NSR e a "severidade dos sintomas de TDAH". Como esperado, ao entrar no segundo passo, apenas a "severidade dos sintomas de TDAH" explicou variância adicional na compreensão de leitura (mudança em $r^{2}$ de $6,4 \% ; p=0,002$ ). A não contribuição da CF e da NSR (todos os $p>0,08$ ) nessa análise é um indício de que a contribuição dessas variáveis para a compreensão de leitura é mediada pela precisão e fluência de leitura (que entraram no primeiro passo).

Por fim, como o TDAH não apresentou contribuição específica para a precisão e fluência de leitura no presente estudo, é possível que o deficit na precisão e/ou fluência de leitura observados em crianças com TDAH se devam a deficit na consciência fonológica e/ou na nomeação seriada rápida. Assim sendo, é importante avaliar essas variáveis em crianças com TDAH, com a finalidade de se prevenir dificuldades mais severas de leitura. Além disso, é importante entender melhor a natureza dos componentes das funções executivas que possivelmente estejam mediando a relação do TDAH com a compreensão de leitura. Por exemplo, Friedman et al. (2017) estimaram o papel do Executivo central por meio da variância comum nas tarefas de memória de curto prazo fonológica e visual. No presente estudo, avaliou-se a atenção seletiva e a flexibilidade cognitiva (que não contribuíram para a compreensão). Então, embora existam evidências de que as funções executivas estejam mediando a relação do TDAH com a compreensão de leitura, isso não indica quais processos exatamente (por exemplo, planejamento, monitoramento, controle inibitório) seriam os responsáveis por essa relação.

\section{CONCLUSÃO}

O objetivo do estudo foi avaliar a relação de variáveis cognitivas e do TDAH com a habilidade de leitura. Foram avaliadas as habilidades fonológicas, a nomeação seriada rápida, o vocabulário, a inteligência e componentes da função executiva. $\mathrm{O}$ presente estudo também considerou o uso ou não de medicação em crianças com TDAH, avançando na caracterização da amostra, na avaliação de diferentes aspectos da leitura e também no controle de diversas variáveis. Os resultados indicaram que a consciência fonológica e a nomeação seriada rápida contribuíram para todos os aspectos da leitura e que o TDAH influenciou de modo negativo na compreensão de leitura. Estes achados contribuem para um melhor entendimento da influência de variáveis cognitivas e do TDAH na precisão, fluência e compreensão de leitura.
Em especial, os resultados do presente estudo ajudam a considerar teoricamente a relação do TDAH com a leitura, pois indicam que a relação do transtorno com a precisão e com a fluência de leitura possa ser de natureza diferente da relação que o TDAH tem com a compreensão de leitura. Nossos resultados indicaram que as principais variáveis explicativas para a precisão e a fluência de leitura foram a consciência fonológica e a nomeação seriada rápida. Portanto, a criança com TDAH que apresenta prejuízo na precisão e na fluência de leitura provavelmente tem prejuízo nesses aspectos da leitura por ter também prejuízos na consciência fonológica e/ou na nomeação seriada rápida. Já em relação à compreensão de leitura, o quadro parece ser diferente, porque o diagnóstico de TDAH continua contribuindo para a explicação dessa habilidade mesmo 
sendo controladas a consciência fonológica e a nomeação seriada rápida. É possível hipotetizar que a relação do TDAH com a compreensão de leitura se deva a um deficit nas funções executivas, o que é comum nessa condição. No entanto, são necessários outros estudos para esclarecer quais processos executivos possam estar especificamente envolvidos nessa relação.

Embora o presente estudo traga uma contribuição importante ao analisar a relação do TDAH com a leitura, considerando diferentes aspectos dessa habilidade e controlando diversas variáveis relevantes, o que ressalta sua contribuição para a área, é necessário considerar suas limitações. Uma delas é que, devido à dificuldade de se encontrar testes normatizados no Brasil condizentes com a faixa etária dos participantes dessa pesquisa, não foram utilizados testes específicos para outros componentes da função executiva, como o planejamento e o monitoramento. Outra limitação foi a impossibilidade de controle das comorbidades existentes nos participantes, em razão de sua heterogeneidade e tamanho da amostra (já que a exclusão de participantes acarretaria a perda de poder estatístico). De qualquer forma, é importante enfatizar que as principais variáveis cognitivas que têm relação com a leitura foram controladas.

\section{REFERÊNCIAS}

Adams, M. J. (1990). Learning to read: Thinking and learning about print. MIT Press.

American Psychiatric Association (2000). Diagnostic and statistical manual of mental disorders: DSM-IV-TR .

American Psychiatric Association. (2014). DSM-5: Manual diagnóstico e estatístico de transtornos mentais. Artmed.

Arnold, T. B., \& Emerson, J. W. (2011) Nonparametric goodnessof-fit tests for discrete null distributions. The R Journal, 3(2), 34-39.

Arrington, C., Kulesz, P., Francis, D., Fletcher, J., \& Barnes, M. (2014). The contribution of attentional control and working memory to reading comprehension and decoding. Scientific Studies Reading, 18(5), 325-346. https://doi.org/10.1080/10 888438.2014 .902461

Baddeley, A. D., \& Hitch, G. (1974). Working memory. In G. H. Bower (Eds.). The psychology of learning and motivation: Advances in research and theory (pp.47-89). Academic Press.

Barkley, R. A. (2002). Transtorno de Déficit de Atenção e Hiperatividade (TDAH): Guia completo e atualizado para os pais, professores e profissionais da saúde. Artmed.

Barkley, R. A. (2005). Critical Issues in research on attention. In G. R. Lyon \& N. A. Krasnegor (Eds). Attention, memory, and executive function (pp.45-56). Paul $\mathrm{H}$. Brookes Publishing.

Bolden, J., Rapport, M. D., Raiker, J. S., Sarver, D. E., \& Kofler, M. J. (2012). Understanding phonological memory deficits in boys with ADHD: Dissociation of short-term storage and articulatory rehearsal processes. Journal of Abnormal Child Psychology, 40(6), 999-1011. https://doi.org/10.1007/s10802-012-9619-6

Bovo, E. B. P., Lima, R. F. Silva, F. C. P., \& Ciasca, S. M. (2016). Relações entre as funções executivas, fluência e compreensão leitora em escolares com dificuldades de aprendizagem. Rev. psicopedag. 33(102), 272-282.

Bowers, P. (2001). Exploration of the basis for rapid naming's relationship to reading. In M. Wolf (Ed.), Dyslexia, fluency, and the brain (pp.41-63). York Press

Bradley, L., \& Bryant, P. (1983). Categorizing sounds and learning to read: A causal connection. Nature, 301(5899), 419-421. https://doi.org/10.1038/301419a0

Cain, K., Bryant, P., \& Oakhill J. (2004). Children's reading comprehension ability: Concurrent prediction by working, verbal ability, and component skills. Journal of Educational Psychology, 96, 31-42. https://doi.org/10.1037/00220663.96.1.31

Cantwell, D. P., \& Baker, L. (1991). Association between attention deficit-hyperactivity disorder and learning disorders. Journal Learning Disabilities, 24(2), 88-95. https://doi. org/10.1177/002221949102400205
Capellini, A. S., Ferreira, T. L., Salgado, C. A., \& Ciasca, S. M. (2007). Desempenho de escolares bons leitores, com Dislexia e com Transtorno de Déficit de Atenção e Hiperatividade em nomeação automática rápida. Revista da Sociedade Brasileira de Fonoaudiologia, 12(2), 114-119. http://dx.doi.org/10.1590/ S151680342007000200008

Coelho, L. F., \& Barbosa, D. L. F. (2012) Intervenção cognitivo comportamental no TDAH. In Muszkat, M., Miranda (Org). TDAH e interdisciplinaridade, intervenção e reabilitação (Vol.1, Cap 6, pp.87-101). All Print.

Correa, J., \& Mousinho, R. (2013). Por um modelo simples de leitura, porém não tão simples assim. In Mota, M. P. E. \& Spinillo, A. (Eds.). Compreensão de textos. (Cap 3, pp. 78-89). Casa do Psicólogo.

Cunha, V. L. O., Silva, C., Lourencetti, M. D. Padula, N. A. M. R., \& Capellini, S. A. (2013). Desempenho de escolares com transtorno de déficit de atenção e hiperatividade em tarefas metalinguisticas e de leitura. Rev. CEFAC. 15, 40 -50. http:// dx.doi.org/10.1590/S1516-18462012005000003

Cutting, L. E., Materek, A., Cole, C. A. S., Levine, T. M., \& Mahone, E. M. (2009). Effects of fluency, oral language, and executive function on reading comprehension performance. Annals of Dyslexia, 59, 34-54. 10.1007/s11881-009-0022-0.

Dancey C. P., \& Reidy J. (2006). Estatística sem matemática para psicologia. Artmed.

Denckla, M. B., \& Rudel, R. G. (1976). Rapid Automatized Naming (R.A.N): Dyslexia differentiated from other learning disabilities, Nuropsychologia, 14(4), 471-479. http://dx.doi. org/10.1016/0028-3932(76)90075-0

DuPaul, J. G., \& Stoner, G. (2007). TDAH nas escolas: Estratégias de avaliação e intervenção. ( $\left.1^{\mathrm{a}} \mathrm{ed}\right)$. (Batista, D. Trans.). M books do Brasil. (Obra original publicada em 2003).

Fergusson, D. M., \& Horwood, L. J. (1992). Attention deficit and reading achievement. Journal of Child Psychology and Psychiatry, 33(2), 375-385. doi: 10.1111/j.1469-7610.1992. tb00873.x

Fonseca, V. da (2008). Cognição, neuropsicologia e aprendizagem: abordagem neuropsicológica e psicopedagógica. Vozes.

Fragoso, A. O., Menezes, A., Dias, N. M., \& Seabra, A. G. (2013). Dificuldade de leitura em crianças com Transtorno de Déficit de Atenção e Hiperatividade: Relato de intervenção com o método fônico. Cadernos de Pós-Graduação em Distúrbios do Desenvolvimento, 13, 14-27.

Friedman, L., Rapport, M., Raiker, J., Orban, S., \& Eckrich, S. (2017). Reading Comprehension in Boys with ADHD: the mediating roles of working memory and orthographic conversion. Journal of Abnormal Child Psychology, 45, 273287. https://doi.org/10.1007/s10802-016-0171-7 
Georgiou, G. K., \& Das, J. P. (2016). What component of executive functions contributes to normal and impaired reading comprehension in young adults? Research in Developmental Disabilities, 49-50, 118-128. https://doi.org/10.1016/j. ridd.2015.12.001

Ghelani, K., Sidhu, R., Jain, U., \& Tannock, R. (2004). Reading comprehension and reading related abilities in adolescents with reading disabilities and attention-deficit/hyperactivity disorder. Dyslexia, 10(4), 364-384. https://doi.org/10.1002/ dys. 285

Gombert, J. (1992). Metalinguistic development. HarvesterWheatsheaf.

Goswami, U., \& Bryant, P. (1990). Phonological skills and learning to read. Lawrence Erlbaum Associates.

Greenhill L. L., Halperin J. M., \& Abikoff, H. (1999). Stimulant medications. J Am Acad Child Adolesc Psychiatry, 38(5), 503-512. https://doi.org/10.1097/00004583-199905000-00011

Grégoire, J., \& Piérart, B. (1997). Avaliação dos problemas de leitura. Artes Médicas.

Holligan, C., \& Johnston, R. S. (1988). The use of phonological information by good and poor readers in memory and reading tasks. Memory e Cognition, 16(6), 522-532. https://doi. org/10.3758/BF03197053

Justi, C. N. G. (2015). A nomeação seriada rápida e o desenvolvimento da leitura: Diferentes hipóteses sobre essa relação. In R. Mousinho, L. M. Alves, \& S. A. Capellini (Eds.), Dislexia: novos temas, novas perspectivas V.3 (pp. 73-82). Wak.

Justi, C. N. G., \& Roazzi, A. (2012). A Contribuição de variáveis cognitivas para a leitura e a escrita no português brasileiro. Psicologia: Reflexão e Crítica, 25(3), 605-614. http://dx.doi. org/10.1590/S0102-79722012000300021

Justi, C. N. G., Roazzi, A., Justi, F. R. R., Henriques, F., \& Cançado, M. (2014). Três hipóteses sobre a natureza das tarefas de nomeação seriada rápida. Psicologia: Teoria e Pesquisa, 30(4) 449-457. https://doi.org/10.1590/S0102-37722014000400010

Kail, R., \& Hall, L. (1994). Processing speed, naming, speed and reading. Developmental Psychology, 20(3), 949-954. https:// doi.org/10.1037/0012-1649.30.6.949

Lobo, P. A. S., \& Lima, L. A. M. (2008). Comparação do desempenho em leitura de palavras de crianças com e sem Transtorno de Déficit de Atenção/Hiperatividade. Rev. CEFAC,10(4), 471483. http://dx.doi.org/10.1590/S1516-18462008000400007

Lúcio, P. S., \& Pinheiro, A. M. V. (2014). Novos Estudos Psicométricos para o subteste de leitura do Teste de Desempenho Escolar. Temas em Psicologia, 22, 109 -119. http://dx.doi.org/10.9788/TP2014.1-09

Lyon, G. R. (1995). Toward a definition of dyslexia. Annals of Dyslexia, 45, 3-27.

Mattos, P., Pinheiro M. A. S., Rohde, L. A., \& Pinto, D. (2006) Apresentação de uma versão em português para uso no Brasil do instrumento MTA-SNAP-IV de avaliação de sintomas de Transtorno do Déficit de Atenção/Hiperatividade e sintomas de Transtorno Desafiador e de Oposição. Rev. Psiquiatr. 28(3), 290-297. http://dx.doi.org/10.1590/S010181082006000300008

Mello, C., Argollo, N., Shayer B., Abreu N., Godinho K., Durán P., ... Miranda, M. (2011). Versão abreviada do WISC-III: Correlação entre QI estimado e QI total em crianças brasileiras. Psicologia: Teoria e Pesquisa, 27(2), 149-155. http://dx.doi. org/10.1590/S0102-37722011000200002

Miranda, A., Mercader, J., Fernández, M., \& Colomer, C. (2017). Reading performance of young adults with ADHD diagnosed in childhood: Relations with Executive Functioning. Journal of Attention Disorders, 21(4), 294-304. https://doi. org/10.1177/1087054713507977

Montiel, J. M. \& Seabra, A. G. (2012). Teoria e pesquisa em avaliação neuropsicológica. Editora Memnom.
Morais, A. G. (2005). O desenvolvimento de habilidades de reflexão fonológica em adultos e jovens pouco escolarizados: Seu papel no aprendizado do sistema de escrita alfabética. In T. F. Albuquerque, E. B. C., \& Leal, T. F. Desafios da educação de jovens e adultos: Construindo práticas de alfabetização (pp. 151-172). Autêntica.

Morais, J., Cary, L., Alegria, J., \& Bertelson, P. (1979). Does awaeeness of speech as a sequence of phones arise spontaneously? Cognition, 4(7), 323-331. http://dx.doi. org/10.1016/0010-0277(79)90020-9

Mousinho, R. (2003). Desenvolvimento da leitura, escrita e seus transtornos. In M. Goldfeld (Ed.). Fundamentos em fonoaudiologia: linguagem (pp. 39-59). Guanabara Koogan.

Mousinho, R., Mesquita, F., Leal, J., \& Pinheiro, L. (2009). Compreensão, velocidade, fluência e precisão de leitura no segundo ano do Ensino Fundamental. Revista Psicopedagogia, 26 (79), 48-54

Oakhill, J., Cain, K., \& Yuill, N. (1998). Individual diferences in children's comprehension skill: Toward na integrated model. In C. Hulme \& R. M. Joshi (Eds.). Reading and spelling develpoment and dosorders (pp. 343-367). Lawrence Erlbaum Associates.

Oakhill, J., Hartt, J., \& Samols, D. (2005). Levels of comprehension monitoring and working memory in good and poor comprehenders. Reading and Writing, 18(7), 657-686.

Oliveira, A. M., Cardoso, M. H. Pinheiro, F. H. Germano, G. D., \& Capellini, S. A. (2011). Desempenho de escolares com Dislexia e Transtorno do Déficit de Atenção e Hiperatividade nos processos de leitura. Revista Brasileira de Crescimento e Desenvolvimento Humano, 21(2), 344-355.

Pastor, P. N., \& Reuben, C. A. (2002). Attention deficit disorder and learning disability?

United States. Vital and health statistics series 10(206), 1-12.

Pastura, G. M. C., Mattos, P., \& Araújo, A. P. Q. C. (2005). Desempenho escolar e transtorno do déficit de atenção e hiperatividade. Revista de Psiquiatria Clínica, 32(6), 324-329. http://dx.doi.org/10.1590/S0101-60832005000600003.

Perfetti, C., \& Hogaboam, T. (1975). Relationship between single word decoding and reading comprehension skill. Journal of Educational Psychology, 67(4), 461-469.

Perfetti, C. A.; Marron, M. A., \& Foltz, P. W. (1996). Sources of comprehension failure: Theoretical perspective and case studies. In C. Cornoldi, \& J. Oakhill (Eds.), Reading difficulties: Processes and intervention (pp. 137-165). Lawrence Erlbaum.

Phelan, T. W. (2005). TDAH - Transtorno de Déficit de Atenção e Hiperatividade: Sintomas, Diagnósticos e Tratamentos. M. Books do Brasil.

Potocki, A., Sanchez, M., Ecalle, J., \& Magnan, A. (2015). Linguistic and cognitive profiles of 8- to 15-year-old children with specific reading comprehension difficulties: The role of executive functions. Journal of Learning Disabilities, 50(2), 128-142. https://doi.org/10.1177/0022219415613080

Puliezi, S., \& Maluf, M. R. (2012). A contribuição da consciência fonológica, memória de trabalho e velocidade de nomeação na aquisição inicial da leitura. Bol. Acad. Paulista de Psicologia, 32(82), 213-227.

Raiker, J. S., Friedman, L. M., Orban, S. A., Kofler, M. J., Sarver, D. E., \& Rapport, M. D. (2017). Phonological working memory deficits in ADHD revisited: The role of lower level information-processing deficits in impaired working memory performance. Journal of Attention Disorders, 1-14. https://doi. org/10.1177/1087054716686182

Salles J. F. (2009). Compreensão de leitura textual entre crianças com e sem dificuldades de leitura e escrita. In: T. Barbosa; C. C. Rodrigues; C. B. Melo; S. A. Capellini; R. Mousinho, \& L. Mendonça. (Eds..). Temas em dislexia (pp.103-113). Artes Médicas. 
Santos, A. A. A. (2004). O Cloze como técnica diagnóstica e remediação da compreensão em leitura. Interação em Psicologia, 8(2), 217-226. http://dx.doi.org/10.5380/psi.v8i2.3257

Santos, A. A. A., Primi, R., Taxa, F., \& Vendramini, C. M. M. (2002). O Teste de Cloze na avaliação da compreensão em leitura, Psicologia: Reflexão e Crítica, 15(3), 549-560. http://dx.doi. org/10.1590/S0102-79722002000300009.

Savage, R., Lavers, N., \& Pillary, V. (2007). Working memory and reading difficulties: What we know and what we don't know about the relationship. Educ. Psychol. Rev., 2(19), 185-221. https://doi.org/10.1007/s10648-006-9024-1

Seigneuric, A., \& Ehrlich, M. (2005). Contribution of working memory capacity to children's reading comprehension: a longitudinal investigation. Reading and Writing, 18(7), 617656. https://doi.org/10.1007/s11145-005-2038-0

Semrud-Clikeman, M., Guy, K., Griffin, J. D., \& Hynd, G. W. (2000). Rapid naming deficit in children and adolescents with reading disabilities and attention deficit hyperactivity disorder, Brain and Language, 74, 70-83. https://doi.org/10.1006/ brln.2000.2337

Seno, M. P. (2010) Transtorno do Déficit de Atenção e Hiperatividade (TDAH): o que os educadores sabem? Rev. Psicopedag. 27(84), 334-343.

Sesma, H. W., Mahone, E. M., Levine, T., Eason, S., \& Cutting, L. (2009). The contribution of executive skills to reading comprehension. Child Neuropsychology, 15(3), 232-246. https://doi.org/10.1080/09297040802220029

Silva, C., Cunha, V. L. O., \& Capellini, S. A. (2011). Desempenho cognitivo-linguístico em leitura de escolares com Transtorno de Déficit de Atenção e Hiperatividade. Rev. Bras. Crescimento Desenvolv. Hum. 21(3), 849-858.

Stein, L., (1994). TDE: Teste de Desempenho Escolar: Manual para aplicação e interpretação. Casa do Psicólogo.

Swanson, H. L. (1999). Reading comprehension and working memory in learning-disabled readers: Is the phonological loop more important than the executive system? Journal of Experimental Child Psychology, 72, 1-31. https://doi. org/10.1006/jecp.1998.2477

Torgensen, J. K. (2000). Individual differences in response to early interventions in reading: The lingering problem of treatment resisters. Learning Disabilities Research and Practice, 15, 55-64.

Van der Leij, A., \& Morfidi, E. (2006) Core deficits and variable differences in dutch poor readers learning english, Journal of Learning Disabilities, 39, 74-90. https://doi.org/10.1177/002 22194060390010701

Wagner, R. K., \& Torgesen, J. K. (1987). The nature of phonological processing and its causal role in the acquisition of reading skills. Psychological Bulletin, 101(2), 192-212. https://doi. org/10.1037/0033-2909.101.2.192

Wechsler, D. (2002). WISC-III: Escala de inteligência Wechsler para crianças [Manual] (3a ed). (Adaptação e padronização brasileira, 1a ed.; Vera Lúcia Marques de Figueiredo). Casa do Psicólogo.

Wolf, M., \& Bowers, P. (1999). The double-deficit hypotesis for the developmental dyslexias. Journal of Educational Psychology, 91(3), 415-438. https://doi.org/10.1037/00220663.91.3.415 